symptomatic cases). An annual register-based Chlamydia screening programme is implemented in three regions since 2008.

Methods The number of persons tested and cases detected in the Chlamydia Screening among 16-29year olds in Amsterdam, Rotterdam and South Limburg, 2008-2010, were compared to consultations and diagnoses in this age group reported in surveillance data from STI centres in the regions and estimates of STI care in general practices in these regions, 2007-2010. Round 3 data are based on the first 6 months of the year.

Results The baseline testing rates (at STI centers and by GP's in year pre-screening) were 10\% in Rotterdam, 13\% in Amsterdam and 6\% in South-Limburg. CSI increased testing rates steeply in the first year to $26-30 \%$ in the cities and $17 \%$ in Limburg; this decreased to $20-21 \%$ and $13 \%$ in round 3 , still doubling testing rates as compared to baseline. Positivity rates at regular STI-care facilities are higher than in CSI: $12-15 \%$ in regular care vs $4-5 \%$ in CSI; therefore the addition of CSI to case-finding in the three regions was lower than that to testing: the screening programme added about $41 \%$ on top of the cases found in regular care in round 1, but this decreased to $20 \%$ in round 3 due to lower participation and positivity rates in consecutive rounds.

Conclusions By comparison to regular testing at STI centers and in general practice, the Chlamydia Screening had a major contribution towards the number of young people tested for Chlamydia in the three regions. The addition towards case-finding was lower, because the case-detection rate of the screening programme was lower than that in regular care. The Screening programme did not seem to affect the number of patients seen in regular care, but double "consumers" cannot be excluded.

\section{P1-S6.13 A NEW APPROACH TO ENCOURAGE HIV TESTING IN HIGH-RISK POPULATIONS AT THE CLINIQUE L'ACTUEL}

\section{doi:10.1136/sextrans-2011-050108.237}

R Thomas, N Machouf, B Trottier, S Vezina, R O'Brien, M Milne, S Lavoie, D Longpré, E Huchet, V K Nguyen. Clinique médicale I'Actuel, Montreal, Canada

Background In Québec it is estimated that 1/3 of those infected do not know their HIV status, that HIV is diagnosed late in $41 \%$, and that sex during primary infection is an important driver of the epidemic. In late 2008 Clinique l'Actuel launched a testing campaign tailored to MSM in Montréal using free rapid tests with the goal of increasing early diagnosis of HIV. In this study we evaluated the feasibility of and potential impact of facilitated access to rapid HIVtesting.

Methods Rapid HIV-tests offered through dedicated clinics were widely advertised in Montréal's MSM community. Patients calling for testing deemed at high risk were given appointments within 2 weeks, where they filled out a short questionnaire, received medical consultation routine STI screening, pre- and post-test counselling and their HIV test results within the hour. Ongoing support, care, and treatment were offered to those testing positive. Results Over 9 months 2500 received HIV testing. 98\% were men and median age was 34 (IOR=26-41). Of these patients, $42 \%$ were new to the clinic, $10 \%$ had never been tested previously, and $29 \%$ had not been tested within the past 2 years. $93 \%$ reported they were more likely to undergo repeat screening because of rapid testing. $2 \%$ were found to be HIV positive. Of these, $60 \%$ cited the rapid test as the primary reason for undergoing screening. 33\% of those testing positive were in primary infection, as compared to $18 \%$ the previous year at Clinique l'Actuel $(p=0.062)$ and $11 \%$ in Québec.

Conclusion Facilitated access to rapid HIV testing can increase uptake in high-risk patients. This may increase early HIV diagnosis and intervention to decrease transmission.

\section{Epidemiology poster session 6: Preventive intervention: Screening: testing P1-S6.14 INTERVENTIONS TO INCREASE RE-TESTING FOR REPEAT
CHLAMYDIAL INFECTION: A SYSTEMATIC REVIEW AND
META-ANALYSIS}

doi:10.1136/sextrans-2011-050108.238

${ }^{1} \mathrm{H}$ Ali, ${ }^{1} \mathrm{R}$ Guy, ${ }^{2} \mathrm{~N}$ Low, ${ }^{3} \mathrm{H}$ Bauer, ${ }^{4} \mathrm{~J}$ Walker, ${ }^{5} \mathrm{~J}$ Klausner, ${ }^{1} \mathrm{~B}$ Donovan, ${ }^{1} \mathrm{~J}$ Kaldor, ${ }^{4} \mathrm{~J}$ Hocking. ${ }^{1}$ National Centre in HIV Epidemiology and Clinical Research, Sydney, Australia; ${ }^{2}$ Division of Clinical Epidemiology and Biostatistics, Institute of Social and Preventive Medicine, University of Bern, Bern, Switzerland, ${ }^{3}$ Program Development and Evaluation, STD Control Branch, California Department of Public Health, USA; ${ }^{4}$ Centre for Women's Health, Gender and Society, Melbourne School of Population Health University of Melbourne, Australia; ${ }^{5}$ Department of Medicine, University of California, USA

Background Repeat infection with Chlamydia trachomatis following treatment is common and increases the risk of sequelae. Despite clinical guidelines recommending re-testing within 3 months of treatment, re-testing rates remains low. We undertook a systematic review of studies which evaluated interventions aimed at increasing re-testing for repeat chlamydial infection.

Methods We searched Medline, EMBASE, trial registries, and conference websites from 2000 to September 2010 using variations of the terms "chlamydia" and "re-testing" and "intervention" to identify studies which compared rates of re-testing for repeat chlamydial infection between patients receiving and not receiving an intervention. We used meta-analysis methods to calculate the overall RR effect on re-testing rates, as well as undertaking a subanalysis by strategy type.

Results We identified eight studies satisfying the inclusion criteria, including four randomised controlled trials and four controlled observational studies. The studies described 12 intervention strategies. All were conducted in the USA. The overall effect estimate RR for any strategy was 1.45 (95\% CI 1.35 to 1.55$)$; $R R=1.80$ (95\% CI 1.63 to 1.97 ) for four studies using reminders such as postcards, phone calls, letters and emails (individually or in combination); 1.25 (95\% CI 1.12 to 1.38 ) for four studies using mailed screening kits with or without reminders; 2.15 (95\% CI 0.92 to 3.37) for two studies using motivational interviewing with or without reminders; 1.35 (95\% CI 0.88 to 1.82 ) for one study promoting re-testing guidelines to clinicians; and 1.16 (95\% CI 0.38 to 1.93) for one study using a $\$ 20$ patient incentive to encourage re-testing.

Conclusion Reminders and mailed screening kits can increase retesting rates by $80 \%$ and $25 \%$ respectively.

\section{P1-S6.15 CHARACTERISTICS AND PREDICTORS OF WOMEN SEEKING RESCREENING FOR STIS AFTER USING THE HTTP:/WWW.IWANTTHEKIT.ORG PROGRAM: WERE THEY INFECTED OR UNINFECTED?}

doi:10.1136/sextrans-2011-050108.239

${ }^{1} \mathrm{C}$ Gaydos, ${ }^{1} \mathrm{Y}$ H Hsieh, ${ }^{1} \mathrm{M}$ Barnes, ${ }^{1} \mathrm{M}$ Jett-Goheen, ${ }^{1} \mathrm{~N}$ Quinn, ${ }^{1} \mathrm{P}$ Agreda, ${ }^{2} \mathrm{P}$ Whittle, ${ }^{1} \mathrm{~T}$ Hogan. ${ }^{1} J o h n s$ Hopkins University, Baltimore, USA; ${ }^{2}$ Baltimore City Health Department, Baltimore, USA

Background CDC recommends rescreening women who are infected with chlamydia (CT) and gonorrhoea (GC) in 3 months. The iwantthekit (IWTK) Internet screening program offered an opportunity to study women who seek rescreening, and determine reported infected status at the previous screening. Mailed IWTK home-collected vaginal swabs are tested for CT, GC, and trichomonas (TV) by NAATs.

Methods Characteristics (demographics, risk behaviours, use perceptions) of repeat users were determined from questionnaires. 
Predictors of repeat users were measured in a matched case-control study by conditional logistic regression analysis. A case $(\mathrm{N}=304)$ was defined as reporting having ever used IWTK before. A control was a user who reported never using the program earlier. Two controls $(\mathrm{N}=608)$ were systematically sampled for each case by matching date of use of IWTK of the case within 3 months.

Results From 2007 to 2010, 17\% of 1747 women who used IWTK for STI testing indicated they had used IWTK previously. Of these, $45 \%$ used it $>2$ times. Mean age was $24.7 \pm 5.7 \mathrm{yr}$; most were African American (69\%); single (87\%); $57 \%$ had 2-4 sexual partners previous yr; $44 \%$ had new partners in last 3 months; $32 \%$ were currently having sex $>1$ person; $16 \%$ practiced anal sex in the last 3 months; $13 \%$ never used condoms; $77 \%$ had been treated for an STI; (5 HIV+). In multivariate analysis, repeat IWTK users were more likely to be $\geq 20 \mathrm{yr}$. (OR=2.10, $95 \%$ CI 1.30 to 3.38$)$ and reside in Maryland ( $\mathrm{OR}=2.03,95 \% \mathrm{CI} 1.31$ to 3.13 ). They were more likely to have had a pelvic exam in past yr $(\mathrm{OR}=2.03,95 \%$ CI 1.36 to 3.05); be treated for an STI ( $\mathrm{OR}=2.32,95 \% \mathrm{CI} 1.57$ to 3.44$)$; to perceive internet screening as confidential $(\mathrm{OR}=1.98,95 \% \mathrm{CI} 1.32$ to 2.97); report results from self-administrated swabs as accurate $(\mathrm{OR}=2.49,95 \% \mathrm{CI} 1.61$ to 3.87$)$; be less likely to drink alcohol before sex $(\mathrm{OR}=0.63,95 \% \mathrm{CI} 0.44$ to 0.91$)$; and to never use condoms with vaginal sex ( $\mathrm{OR}=0.43,95 \% \mathrm{CI} 0.27$ to 0.69$)$. Of repeat users, $84.2 \%$ reported having a negative prior test and 48/304 (15.8\%) reported last test positive; 27 had CT; 24 had TV; 3 had GC; 6 were mixed infections. At present test, 40 (13.2\%) were positive: 14 had CT, 2 had GC, 28 had TV; 4 were mixed infections. Previous TV was associated with current TV positivity $(p<0.05)$.

Conclusions IWTK attracted many previous participants who practiced high-risk sexual behaviours to use IWTK for repeat STI testing. IWTK may offer an alternate approach for rescreening previously infected women.

\section{P1-S6.16 AUSTRALIAN CHLAMYDIA CONTROL EFFECTIVENESS PILOT: PRELIMINARY RESULTS FROM A TRIAL OF CHLAMYDIA TESTING IN GENERAL PRACTICE}

\section{doi:10.1136/sextrans-2011-050108.240}

${ }^{1} \mathrm{~J}$ Hocking, ${ }^{1} \mathrm{M}$ Temple-Smith, ${ }^{1} \mathrm{~S}$ Poznanski, ${ }^{2} \mathrm{R}$ Guy, ${ }^{3} \mathrm{~N}$ Low, ${ }^{2} \mathrm{~B}$ Donovan, ${ }^{1} \mathrm{~J}$ Gunn, ${ }^{2} \mathrm{M}$ Law, ${ }^{2} \mathrm{~J}$ Kaldor, ${ }^{1} \mathrm{C}$ Fairley. ${ }^{1}$ University of Melbourne, Carlton, Australia; ${ }^{2}$ University of New South Wales, Australia; ${ }^{3}$ University of Bern, Switzerland

Background While opportunistic chlamydia screening is conducted in several countries, debate remains about the effectiveness of population-based screening programs for reducing chlamydia transmission and its morbidity. The Australian Chlamydia Control Effectiveness Pilot (ACCEPt) aims to assess the feasibility, acceptability, efficacy and cost-effectiveness of annual chlamydia testing in general practice.

Methods ACCEPt is a cluster randomised controlled trial targeting sexually active 16-29-year old women and men for annual chlamydia testing. 54 postcodes (towns) are being randomised and all GP clinics within each area invited to participate. A multifaceted intervention to maximise testing includes: a computer alert prompting GPs to test; incentive payments for GPs and payments for employing practice nurses; a recall system to encourage annual testing; partner notification, and; information/support with regular feedback on testing performance. Clinics in the control group are encouraged to continue their usual practice. The intervention will be in place for up to 4 years. The primary outcome is change in chlamydia prevalence among a consecutive sample of $80-100$ patients attending participating clinics in each postcode (total sample size about 4500) measured at the beginning and end of the trial. Secondary outcomes include pelvic inflammatory disease and chlamydia testing rates.
Results Recruitment began in July 2010 and 282 GPs in 69 clinics in 24 postcodes have been recruited to date in the States of Victoria, New South Wales and Queensland. Four clinics have refused so far and these postcodes have been excluded. To date, 61516 to 29 year olds have been tested during the baseline prevalence survey with a participation rate of $70 \%$. Overall chlamydia prevalence is $4.0 \%$ (95\% CI $2.5 \%$ to $6.0 \%$ ). Prevalence is slightly higher among males (4.5\%; $95 \%$ CI $2.0 \%$ to $8.7 \%$ ) than females $(3.7 \%$; $95 \%$ CI $2.0 \%$ to $6.3 \%, p=0.7)$ and in rural (6.9\%; $95 \%$ CI $3.8 \%$ to $11.2 \%)$ compared with metropolitan areas (2.2\%; 95\% CI $0.9 \%, 4.4 \%, \mathrm{p}<0.01)$. Recruitment will be completed by December 2011 with the intervention period running till end of 2014 .

Conclusions This study shows high participation rates by GP clinics and by individuals invited to take part in the prevalence survey. Results will determine whether annual chlamydia testing is effective at reducing transmission and morbidity and will inform the optimal design of a chlamydia testing program in Australia.

\section{P1-S6.17 OPTING OUT TESTING FOR HIV IN DUTCH STI CLINICS: DOES IT WORK?}

doi:10.1136/sextrans-2011-050108.241

${ }^{1} \mathrm{~F}$ Koedijk, ${ }^{2,3} \mathrm{~J}$ van Bergen, ${ }^{4,5} \mathrm{~N}$ Dukers, ${ }^{4,5} \mathrm{C}$ Hoebe, ${ }^{3,6} \mathrm{M}$ van der Sande, ${ }^{7}$ on behalf of the Dutch STI centres. 'National Institute of Public Health and the Environment, Bilthoven, Netherlands; ${ }^{2}$ STI AIDS Netherlands, Amsterdam, the Netherlands; ${ }^{3}$ Centre for Infectious Diseases and Control, National Institute for Public Health and the Environment, Bilthoven, the Netherlands Netherlands; ${ }^{4}$ Department of Infectious Diseases, Public Health Service South Limburg; ${ }^{5}$ Department of Medical Microbiology, Maastricht University Medical Center, Maastricht, the Netherlands Netherlands; ${ }^{6}$ Academic Medical Centre Utrecht, Utrecht, the Netherlands Netherlands; ${ }^{7} A$ van Daal, $P$ van Leeuwen, F de Groot, A Niekamp, M Langevoort, A van Camerijk, J van de Sande, E van der Veen Netherlands

Background In 2005, STI centres in the Netherlands started provider-initiated HIV testing policy, in order to decrease the proportion of people unaware of their positive HIV status and to enable interruption of transmission and create more opportunities for timely treatment. This policy gradually evolved towards opt-out HIV testing and in January 2010, this became the official policy within all Dutch STI centres. The effects of the change in HIV test policy were studied and factors associated with opting out for HIV testing were identified.

Methods Data from January 2004 to June 2010 from 488727 consultations registered in the Dutch national surveillance in the STI centres were used to characterise current practices on HIV testing. Known HIV positives were excluded from analyses. Logistic regression analyses were done separately for men having sex with men (MSM) and heterosexuals, to identify factors associated with refusing an HIV test.

Results Since 2004, the percentages of HIV testing within an STI consultation have increased significantly from $56 \%$ up to $92 \%$ in 2009 , and further to $97 \%$ in the first half of 2010 when opting out was implemented nationally (both $\mathrm{p}<0.001$ ). STI were significantly more often diagnosed in clients not tested on HIV during their consultation $(p<0.001)$, except in 2010. Using 2010 data, MSM being older than 25 years (OR: 1.8, 95\% CI 1.2-2.6), those having STI symptoms (OR $2.295 \%$ CI 1.7 to 2.8 ) and those with a previous STI (OR: $1.5,95 \%$ CI 1.2 to 2.0 ) more often refused an HIV test. For heterosexuals, having had a previous STI (OR: 1.6, 95\% CI 1.3 to 2.0 ), being female (OR: $1.2,95 \%$ CI 1.0 to 1.4 ) and being younger than 25 years (OR: $1.2,95 \%$ CI 1.0 to 1.4 ) were independent factors associated with refusing an HIV test.

Conclusions Although provider-initiated HIV testing already increased HIV testing rates, national implementation of opting out for HIV testing increased this uptake even more. Standard testing on HIV in every STI clinic is shown to be highly feasible and effective. 